\title{
Pharmacist Intervention in Fluvoxamine Induced Alopecia: A Case Report in Tertiary Care Teaching Hospital
}

\author{
Benson Koshy ${ }^{1 *}$, Shashank S Hosur ${ }^{1}$, Stimson Jose ${ }^{1}$, Yogananda Rajashekarachar ${ }^{1}$, Gopal Das ${ }^{2}$ \\ ${ }^{1}$ Department of Pharmacy Practice, SJM College of Pharmacy, Chitradurga, INDIA. \\ ${ }^{2}$ Department of Psychiatry, Basaveswara Medical College Hospital \& Research Centre, Chitradurga, INDIA
}

\begin{abstract}
Hair loss (alopecia) is an occasional side effect associated with psychotropic drugs use. Fluvoxamine is a synthetic antidepressant which acts as a selective serotonin reuptake inhibitor as well as sigma-1 receptor agonist. Psychotropic drugs are considered to cause hair loss by affecting the telogenic phase of hair follicle. Here we present a case of a 28 year old male patient reported with diffuse hair loss after administration of fluvoxamine and condition remitted after cessation of the drug. Our report suggests the possibility of developing alopecia with fluvoxamine use.
\end{abstract}

Key words: Fluvoxamine, Adverse effect, Alopecia, Naranjo Causality assessment scale, Drug intervention, Clinical improvement.

\section{INTRODUCTION}

Hair loss is not a common side effect of psychotropic drugs. ${ }^{1}$ The most related psychotropic drug class with this side effect is mood stabilizers. ${ }^{2}$ Selective serotonin reuptake inhibitors (SSRI's) are commonly prescribed psychotropic medication due to its better overall safety and tolerability than older antidepressants ${ }^{3}$. It is used in conditions like major depressive disorder, obsessive compulsive disorder, panic disorder, generalized anxiety disorder, posttraumatic stress disorder and many other psychiatric disorders ${ }^{4}$. But studies regarding SSRI's induced alopecia are limited in number. Drug induced hair loss generally progresses with no scars and improves after quitting the drug. When a drug is suspected to induce hair loss, other etiological causes including hyperthyroidism, hypothyroidism, and trichotillomania associated with other psychiatric disorders, hormonal imbalances as well as iron copper and zinc deficiency and medications like anticonvulsants, antihypertensive agents, anticoagulants, non-steroidal, antiulcer drugs and anti-inflammatory drugs should be considered in differential diagonosis ${ }^{5}$.

\section{CASE REPORT}

A 28 year old, married male businessman presented to psychiatric out-patient department of Basaveshwara Medical College Hospital \& Research Centre (BMCH \& RC) with chief complaints of lack of confidence, guilt feelings and losing interest in pleasurable activities and making frequent errors in his daily life. He also reports of excessive worries about his job, future and difficulty in relaxing even at his leisure time which makes his life stressful.

Complete medical history of the patient which presents no medically significant information and no substance or drug abuse. The diagnosis was performed as per DSMIV criteria as Major Depressive Disorder (MDD). Initially Fluvoxamine was started at a dose of $50 \mathrm{mg} /$ day as treatment for MDD. 3 weeks later, the patient returned with
DOI: 10.5530/ijopp.9.2.14

Address for correspondence: Benson Koshy, S J M College of Pharmacy, JMIT Campus, NH-4 Bypass, Chitradurga-577502, INDIA. Contact no: +919447444315

E-mail: benzkoshy@gmail.com

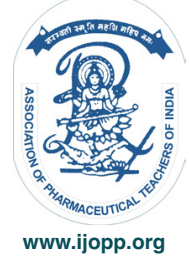


a complaint of active hair loss, hence the patient was advised to consult the dermatology department. Also as per advice thyroid, liver and kidney function tests were performed and serum electrolytes, complete urine analysis, iron, folate, zinc and copper levels were analyzed and results were normal. Hence, the patient was kept under further observation for 3 days. At his next out-patient visit for review, it was observed that the hair loss was still progressing even though depressive symptoms were found to be decreased. Later the clinical pharmacist used the adverse drug reaction scale to assess the severity of the reaction as per Psychiatrist and Dermatologist advice. Naranjo et al. Adverse Drug Reaction Probability scale was applied and a score of 6 was obtained, which determined the alopecia as a probable adverse effect of drug. Since medical examinations also couldn't rule out other reasons for the hair loss, conservatively it was considered to be caused by the therapy. Hence fluvoxamine was advised to discontinue and desvenlafaxine was prescribed. After an observation period of 15 days, hair loss was found to be gradually reduced. During his next visit after 1 month, clinical global impression rating scale was used to assess the symptoms severity and treatment efficacy and a score of was 2.1 (much improved) was obtained. The patient had no further complaints of hair loss.

\section{DISCUSSION}

Studies reporting SSRI's induced alopecia are very limited in number.

Spigset o et al. reported that $11.4 \%$ of all SSRI's induced side effects are dermatological and were more common with fluoxetine use; and the most common was rash. ${ }^{6}$ Several well controlled studies have confirmed the efficacy of fluvoxamine in children and adolescents with OCD, SAD and other anxiety disorders. Many medications can cause hair loss, but the mechanism has not been understood completely, ${ }^{7}$ but is considered to be caused by influencing the telogen phase of hair production. ${ }^{8}$ There are other antidepressant medications too that may cause telogen hair loss. Fluoxetine is the most common SSRI to induce alopecia. ${ }^{9}$

If a patient complaints of hair loss after taking a drug, all other pathological reasons should be taken into consideration. A gentle hair pull test will be performed by a dermatologist to confirm hair loss. It is not easy to determine whether hair loss is due to drug use since there are no specific diagnostic criteria. For diagnosing drug-induced alopecia, other possible etiological causes should be considered, the hair loss with respect to the drug starting time or dose changes should be considered. ${ }^{10}$ A reliable way to verify the diagnosis of drug induced alopecia is the finding of hair loss reduction or disappearance after the cessation of drug and the relapse with restarting the drug. ${ }^{11}$ In this case, fluvoxamine was discontinued after the hair loss was confirmed and was not given further. The hair loss was considered to be induced by fluvoxamine use, because there exist a temporal relationship between hair loss and drug starting time: hair loss disappeared when the drug was stopped.

\section{CONCLUSION}

Our report suggests the possibility of developing alopecia with fluvoxamine which was a possible ADR as per Naranjo et al. Adverse Drug Reaction Probability scale. Therefore, further studies are required to confirm the mechanism and risk factors associated with fluvoxamine induced alopecia.

\section{ACKNOWLEDGEMENTS}

We are thankful to Principal and Head of the Department of Pharmacy Practice of SJM college of Pharmacy for providing necessary facilities and support. We would also sincerely acknowledge the guidance provided by Senior Psychiatrist, Basaweshwara Medical College and Research centre, Chitradurga, Karnataka, INDIA.

\section{CONFLICT OF INTEREST}

The authors have no conflicts of interest.

\section{REFERENCES}

1. Mercke $\mathrm{Y}$, Sheng $\mathrm{H}$, Khan T, Lippmann S. Hair loss in psychopharmacology. Annals of Clinical Psychiatry. 2000;12(1):35-42.

2. Kuloglu M, Korkmaz S, Kilic N, Saglam S, Giirok MG, Atmaca M. Olanzapine induced hair loss: a case report. Bulletin of Clinical Psychopharmacology. 2012;22(4):362-5.

3. Ferguson JM. Antidepressant Medications: Adverse Effects and Tolerability. Prim Care Companion J Clin Psychiatry. 2001;3(1):22-7.

4. Kaplan HI, Sadock BJ. 'Synopsis of Pschiatr). Behavioral Sciences/Clinical Psychiatry, Lippincott $8^{\text {th }}$ edition. 1998.

5. McLean RM, Harrison-Woolrych M. Alopecia associated with quetiapine. International Clinical Psychopharmacology. 2007(2):117-9.

6. Spigset $O$. Adverse reactions of selective serotonin reuptake inhibitors: reports from a spontaneous reporting system. Drug Safety. 1999;20(3):277-87.

7. Irons J. Fluvoxamine in the treatment of anxiety disorders. Neuropsychiatr Dis Treat. 2005;1(4):289- 99.

8. Mercke, Sheng H, Khan T, Lippmann S. Hair loss in psychopharmacology. Annals of Clinical Psychiatry. 2000;12(1):35-42.

9. Gautam M. Alopecia due to psychotropic medications. Annals of Pharmacotherapy. 1999;33(5):631-7.

10. Ogilvie AD. Hair loss during fluoxetine treatment. The Lancet 1993; 342(8884):1423.

11. Hillmans K, Blume-Peytavi U. Diagnosis of hair disorders. Seminars in cutaneous medicine and surgery. 2009;28(1):33-8. 